\title{
Reviewer Acknowledgements for Global Journal of Health Science, Vol. 10, No. 4
}

Global Journal of Health Science wishes to acknowledge the following individuals for their assistance with peer review of manuscripts for this issue. Their help and contributions in maintaining the quality of the journal are greatly appreciated.

Global Journal of Health Science is recruiting reviewers for the journal. If you are interested in becoming a reviewer, we welcome you to join us. Please find the application form and details at http://recruitment.ccsenet.org and e-mail the completed application form to gjhs@ccsenet.org.

\section{Reviewers for Volume 10, Number 4}

Abiodun Adeniran, University of Ilorin, Nigeria

Ama Pokuaa Fenny, University of Ghana, Ghana

Angus N Oli, Nnamdi Azikiwe University, Nigeria

António Calha, Polytechnic Institute of Portalegre, Portugal

Ayesha Johnson, University of South Florida, United States

Basak Baglama, Near East University, Cyprus

Chung-Yu Chen, Kaohsiung Medical University Hospital, Taiwan, Province of China

Dalia Salah El-Deen El-Sedawy, Cairo University, Egypt

David John Lindsay, James Cook University, Australia

Domitila Augusta Huber, Federal University of Santa Catarina, Brazil

Evangelia Mavrikaki, National \& Kapodistrian University of Athens, Greece

Evanthia Sakellari, Technological Educational Institute of Athens, Greece

Fernanda Barbosa Lima-Christian, Federal University of Santa Catarina, Brazil

Gunta Beta, Rī ga Stradiņš University, Latvia

Horng-Jyh Tsai, Kuang Tien General Hospital, Taiwan, Province of China

Jason J W Tsai, Lincoln College, United Kingdom

José Joaquín Mira, Universidad Miguel Hernández, Spain

Loray Daws, British Columbia Masterson Institute, Canada

Meng Zhao, Texas A\&M University at Corpus Christi, United States

Mini Sood, Monash University Malaysia, Malaysia

Pi-Ming Yeh, Missouri Western State University, United States

Robert Sloan, Kagoshima University Graduate School of Medical and Dental Sciences, Japan

Roger Ho, National University of Singapore, Singapore

Sara Melo, Queen's University Belfast, United Kingdom 